Encephalography is a procedure in which air is injected by the more convenient lumbar subarachnoid route. This method has been less extensively employed on the grounds that the air does not enter the ventricular system so satisfactorily, and that the attendant risk is greater, but it may be most informative and is practically free from danger if the correct technique is utilized. It should not be attempted if the intracranial pressure is increased.

Arterial encephalography-radiography of the cerebral arteries after the injection of thorotrast into the internal carotid in the neck-was introduced in 1931 by Moniz, who affirms that the abnormalities in the course and distribution of the arteries so demonstrated afford as useful information as does ventriculography, and that the procedure is quite free from risk.

\section{The Present Position}

A comparison of the operative results of to-day with those of the past necessitates a consideration of statistical data ; and here we owe a special debt of gratitude to Harvey Cushing, the pioneer of the surgery of brain tumour, who has from time to time during the past three decades published his operative results. In his communication at the International Neurological Congress at Berne in 1931, entitled "The Surgical-Mortality Percentages pertaining to a Series of Two Thousand Verified Intracranial Tumours," Cushing analyses his unique experience. His figures are the more impressive since every death in hospital from any cause whatsoever, no matter how long the interval after operation, is regarded as a post-operative fatality. The facts, as the following table shows, are very striking.

Comparison of Operative Mortality Percentages for Verified Tumours of Four Major Groups Divided in Three Periods

\begin{tabular}{|c|c|c|c|}
\hline & $\begin{array}{c}\text { Hopkirs Series } \\
\text { to } 1912\end{array}$ & $\begin{array}{c}\text { Brigham Series } \\
\text { to } 1529\end{array}$ & $\begin{array}{l}\text { July, 1928, to } \\
\text { Juiy, } 1931\end{array}$ \\
\hline Gliomas (varia)... & $\begin{array}{c}\text { per cent. } \\
30.9\end{array}$ & $\begin{array}{c}\text { per cent. } \\
17.8\end{array}$ & $\begin{array}{c}\text { per cent. } \\
11.0\end{array}$ \\
\hline Pituitary adenomas ... & 13.5 & 5.3 & 5.7 \\
\hline $\begin{array}{lll}\text { Meningiomas } & \ldots & \ldots\end{array}$ & 21.0 & 10.3 & 7.7 \\
\hline Acoustic tumours & 25.0 & 11.5 & 4.4 \\
\hline
\end{tabular}

Cushing points out that the improvement in the results is not due to a selection of relatively" favourable lesions, for " the proportion of patients admitted as "forlorn hopes ' in the terminable stages of their malady, often after ill-judged procedures at the hands of surgeons with little or no neuro-surgical training, is as large as ever it was." The principal steps to which the more favourable results are to be attributed he enumerates chronologically as follows: (1) the generally accepted methods of decompression to relieve tension; (2) such irreproachable woundhealing that secondary infections are practically unknown ; (3) the separate closure of the galea by buried fine black silk sutures, which has caused the once dreaded fungus cerebri to be well-nigh forgotten ; (4) the introduction by de Martel, in place of ether inhalation, of local anaesthesia, now supplemented, when necessary, by the rectal administration of tribromethanol ; (5) the more precise tumour localization which in obscure cases Dandy's ventriculography permits us to make; (6) the use of a motordriven suction apparatus as an indispensable adjunct to every operation; and (7) the successive improvements in methods of haemostasis which since 1927 have been most advantageously supplemented by the introduction of electro-surgical devices. But the operation, he adds, is by no means the whole story. After-care is equally important, for unsuspected complications may arise.
Patients are not removed from the operating suite until the danger of a post-operative clot has passed. Many lives, too, he states, have undoubtedly been saved by the provision of a highly trained nurse, who may be expected to appreciate " the significance of symptoms which indicate that something is wrong with a patient recently operated upon for a brain tumour ; and a few hours' delay due to the misinterpretation or neglect of a warning signal may mean the difference between a fatality and a recovery."

The operative risks in cases of intracranial tumour have been very materially reduced, and successful removal is now a commonplace. Surgical technique has indeed made great progress, and yet Cushing writes: "In another thirty years our present operative procedures will doubtless appear as crude to our successors as do those of thirty years ago to us." But while the operative results in the case of the extracerebral and encapsuled growths are so much more satisfactory, the same cannot be said of the infiltrating tumours. Some form of treatment is called for which will inhibit the reproduction of the neoplastic tissue and leave unscathed the functional elements of the nervous system. Deep $x$-ray therapy, which has been extensively employed of recent years, has an undoubtedly beneficial effect upon some forms of tumour, and notably upon the pituitary adenomata and some of the gliomata. This form of treatment is, however, still in the experimental stage, and time must elapse to permit of definite conclusions as to its efficacy and limitations. It is, too, a dangerous procedure, and should only be applied by those who are fully conversant with the method and its risks.

\section{THE TREATMENT OF CHRONIC SUPPURATIVE OTITIS MEDIA* By}

L. GRAHAM BROWN, M.D., F.R.C.S.

SURGEON, EAR, NOSE, AND THROAT DEPARTMENT, CHARING CROSS HOSPITAL

The successful treatment of chronic middle-ear suppuration remains one of our greatest problems. The reason is not far to: seek when we consider the tortuous anatomy of the middle-ear cleft ; our frequent inability by clinical methods to define accurately the nature and extent of the pathological lesion; the difficulty often confronting us when we attempt to eradicate the disease by surgical or other means of conserving the function of hearing; and the risks that may befall the patient. The latter concerns not only his hearing, but even his life if we delay too long a surgical intervention for which the necessity is not always plainly indicated.

Further, we may still have to encounter the indifference of a public that continues to regard a chronically discharging ear as merely a tiresome, if at times a somewhat distressing, complaint, and do not rightly appraise its grave potentialities as we see them-namely, at best a permanent impairment of hearing (and therefore a loss of both individual and national efficiency), or, maybe, the ever-impending disaster of an intracranial affection. For the latter reasons alone, apart from any feelings of pride in our specialty, it behoves us, in an age when such great scientific progress has been made in other fields of medicine and surgery, to devote our fullest energies towards a solution of this important problem.

* Read in opening a discussion in the Section of Oto-RhinoLaryngology at the Annual Meeting of the British Medical Association, Melbourne, 1935. 


\section{Prophylaxis}

It will be our first duty continually to emphasize the methods of prevention of chronic middle-ear suppuration. These consist in the already well-established rules of guidance in diminishing the possibilities of aural infection, its proper care and treatment in the acute stage-especially when complicating the exanthematous fevers-and, in those cases tending to become chronic, searching for and dealing with a cause in the nose or nasopharynx, or possibly in the mastoid itself. These methods are too well known to be described further, and much has already been accomplished in this respect by the general practitioner, public bodies, and hospital institutions.

What is not yet generally known and appreciated, however, is the importance of always bearing in mind the possibility of a latent mastoiditis, especially in the case of young children. This, if not recognized and dealt with surgically, may prove a putent causative element in the establishment of a chronic suppuration. It has surely been the experience of many to have opened up a mastoid cortex and found a large area of infection in the cells and antrum, when clinically there has been very little evidence of this. In such cases efficient radiograms are, in my opinion, of great value, and may ass:st in making up our minds to intervene in the case of the acute suppurating ear that is tending to become chronic. In fact, one may stress this point further, and say that any case of acute otitis media that does not resolve, under appropriate treatment, after the normal period of ten to fourteen days-other exciting causes in the nose and nasopharynx being absent-should be viewed with suspicion and considered as a possible case of latent mastoiditis. If operation is decided upon the simple form of mastoidectomy is then indicated, and proves no very serious matter when, as I shall describe later, modern methods of surgical technique and aftertreatment are employed.

\section{Aim of Treatment}

Obviously the aim of treatment is to get rid of the infective focus that is keeping up the chronic inflammatory discharge, and at the same time to attempt to preserve whatever useful degree of hearing remains. Unfortunately, these two objects tend to conflict, especially when we have to employ surgical methods of treatment. Hence comes our des:re to employ conservative methods as much as possible, and our reluctance to undertake operative measures, except in cases of emergency when grave complications threaten, or, as a last resort, when conservative methods have plainly failed. The former ideal-namely, to cure all cases oy conservative medical measures-has made us try a great variety of direct methods of attack, mostly via the external auditory meatus. These are legion, and range from simple cleansing solutions, medicated drops, and solutions with varying degrees of disinfecting, digesting, penetrating, and bactericidal power, and powders with similar properties, to elestrical methods, such as that of zinc ionization, electrolysis, diathermy, light therapy, and even the application of $x$ rays. All of us have had our successes in this respect, and our zeal to persist in this line of treatment is commendable ; moreover, it is doubtless responsible for the enthusiasm with which fresh products are being continually brought on to the market and have their vogue-at least for a time.

However, in a large number of cases we are reluctantly forced to admit failure. The discharge continues, or at best is limited for a time and then recurs. We say we fail because the area of disease is inaccessible to our cleansing lotions, medicated flu:ds, etc. Then begin a series of minor surgical attacks upon the area of disease, consisting of incisions of the drum membrane, removal of polypi, curetting of granulations, and even of the underlying bony infection and the removal of ossicles. By such procedures we admit that not only are the areas of disease made more accessible to our applications, but better drainage of the discharge is thereby established. A further percentage of cures is thus effected, but failures still remain, and in these we continue to say that the disease cannot properly be reached. We are now confronted with the choice of letting well alone, or of undertaking a major surgical operation upon the mastoid bone itself. We have, moreover, to decide whether a simple mastoidectomy will suffice, or whether we should extend this to deal with the contents in part or whole of the middle-ear cavity, and thus perform the conservative radical or the complete radical mastoid operation.

Our indications for operation will be partly gathered from a careful study of the general symptoms of the disease, the clinical appearance of the middle ear, as viewed through the external auditory meatus, the chronicity and effects of previous forms of conservative medical treatment, and, lastly, and by no means least important, the degree of useful hearing that remains. In some cases our indications for opening up the mastoid bone will be defined clearly, but others will occur in which it will not always be possible definitely to judge the efficacy of any particular form of operation. Our clinical methods of examination do not yet allow of our gauging correctly the exact nature and extent of a pathological process in the middle-ear cleft, and even skiagrams give, in most cases, but an imperfect shadow of the reality. Hence I maintain that only upon opening up the mastoid antium, removing the posterior bony wall of the meatus, the bridge of bone over the aditus, and part of the outer attic wall can we obtain a real picture of the extent of the lesion present. This procedure will serve, not only when we have clear indications for operation, but also in those cases where we have decided upon operation but are not yet certain in our judgement from the clinical evidence available as to what type of mastoid operation wo should perform.

In view of the great importance of preserving whatever useful hearing remains, let me urge the following dictumnamely, the more radical the method of surgical procedure on the ear that tends to destroy the usefulness of the hearing function the more precise should be its indications.

\section{Conservative Medical Methods of Treatment}

Our policy of treating chronic suppuration by the most conservative measures has led to the employment of a vast number of different medicaments, to which $I$ have already referred and which are said to owe their success to their penetrating, disinfecting, or bactericidal power. It is difficult to assess the real value of any one of them, for we are as yet unable to prove how far they penetrate or how potent they are in antiseptic or bactericidal properties when used in concentrations which are themselves non-irritating to the living tissues; for it is upon the vitality of the latter that we rely for repair of the lesion. Hence I am sceptical enough to disbelieve in their curative value per se, but inclined rather to consider them as useful factors in providing the best possible conditions for promoting drainage and, to sothe extent, aeration of the affected area of the lesion.

In a word, until we can correctly standardize their properties and actions we must look upon them as merely cleansing and mildly disinfectant methods of treatment, and give to Nature herself the credit of effecting a cure, owing to adequate drainage of toxic secretions and the healing power of the tissues. In so far as Nature fails in this latter respect, and there is mechanical obstruction to efficient drainage, so will the chronicity of the affection prevail. However, to the advocate of this or that method of conservative medical treatment his want of success in any given case is rather attributed to the inaccessibility of 
the affected area to his chosen medicament. The fact remains that in a total number of cases of chronic suppuration treated carefully by one or other of these methods it can fairly be claimed that a percentage of 70 to 75 of cases result in cure.

Our clinical study of these successful cases has taught us the following facts. "The lesion is generally confined to the atrium of the middle ear. The perforation is in the majority of cases central and not marginal, and may be small or large. Recurrence of suppuration is apt to occur following a cold or after bathing, and in the presence of enlarged tonsils and adenoids or disease of the nasal accessory sinuses. Hence.the importance of dealing with these contributory factors. Small perforations may close spontaneously, but if they persist attempts should be made to close them by means of trichloracetic acid, chromic acid, or silver nitrate carefully applied to their margins. Large perforations cannot be closed in this manner, but some may do so spontaneously by forming adhesions to the inner wall of the tympanum, while the remainder may persist indefinitely, but give rise to no more than a thin, scanty mucous secretion. This last class may be considered " safe," and any possible advantage that may be gained in securing a dry ear by subjecting them to the radical mastoid operation will doubtless be equally offset by the resultant depreciation of hearing.

It may be fairly asked how long one should persist in medical forms of treatment before deciding upon some surgical procedure. It is admittedly a difficult question to answer, and especially in those long-standing adult cases where the disease through time may have become firmly established, and, moreover, may have affected the bony as well as the membranous structures. Which of us, however, has not seen, even in these cases, a miraculous cure result after a process of careful cleansing and the application of one's own favourite disinfectant? Also, this teaches us not to rest content with any one particular form of medical treatment, but to ring the changes on them just as we are wont to do in most kinds of empirical treatment. Since most of us, however, have predilections for some particular form of treatment I shall now state my own, confessing at the same time my readiness to change over to others when I consider in any particular case my own has become ineffective.

\section{Author's Method}

For cleansing purposes I prefer the wet method to the dry. It is a simple method, which has withstood the test of time, and consists in the instillation of hydrogen peroxide drops into the meatus, followed by syringing with boric acid solution. I cannot agree with those who say that syringing is a dangerous process. In the whole of my experience I have never seen any harm result, provided it is carried out with reasonable care and with due regard to the comfort of the patient. On the contrary, I know of no more effective method of cleansing a meatus, and none which causes less anxiety and discomfort to the patient, especially the infant and small child. Subsequently, as a disinfectant, I recommend the instillation of guttae s.v.r. (70 per cent.) or, upon occasions-especially when there are granulations present on the inner tympanic wall-the application of pulv. a.b. +1 per cent. iodine. In adults I am prepared to continue this form of medical treatment, no urgent reasons for operation supervening, for a period of two to three months before advising on the next stage of procedure, which by that time will surely have been suggested by the endoscopic appearances of the lesion. In children, however, the problem is not quite the same, since the preservation of their hearing throughout their early educative life is most important, and places an added responsibility upon us. If there is not a quick response to treatment, say in a period of one to two months, and this of course includes a diminution of, and alteration in, the character of the discharge, and, better still, after a trial of Friel's method of zinc ionization, and any exciting causes in the nose and nasopharynx are proved absent, resort should then be had to some form of surgical treatment.

\section{Minor Operative Measures}

We estimate that 25 to $30^{\circ}$ per cent. of all cases require some form of operative treatment. In accordance with our stated policy of proceeding on conservative lines we first employ minor operative methods, in the hope of promoting better drainage and allowing accessibility of antiseptics to the diseased areas. These minor surgical interventions may be briefly stated. They consist in incision of the tympanic membrane, when the existing perforation is small and the membrane reddened, thus indicating retention of pus; the removal of polypi or granulations ; very occasionally, the curetting of a fistulous tract into the meatus; or even, in some cases, ossiculectomy.

The results of such treatment are, in our experience, on the whole unsatisfactory, and in the best conditions can be stated to effect a cure in about 5 per cent. of all such cases. Granulations, except those originating from the mouth of a fistulous tract into the meatus, are difficult to remove safely, polypi are more often than not prone to recur, and the removal of necrosed ossicles via the meatus does not usually get rid of a deeper-seated lesion such as the necrosed ossicles signify. However, such operations may be considered justifiable, first, in the hope that they will bring about the occasional cure, and, secondly, as an expedient when other more radical measures of operation are refused.

\section{Major Operative Measures}

This part of our subject is worthy of the most serious consideration. We have to determine the correct procedure in the 20 per cent. of all cases that require an operation on the mastoid bone. Moreover, we have to decide on the type of operation that will best suit the individual case and that will be neither too limited to deal adequately with the pathological site of the lesion nor too extensive to effect this and at the same time not diminish unduly a useful function of hearing. If the hearing is at all useful attempts should be made to preserve it. This applies even in some cases of cholesteatoma. The preservation of the hearing function demands as little interference as possible with the membranous meatus and the contents of the middle ear. The simple or Schwartze mastoid operation fulfils best this latter purpose, but its indications are unfortunately limited, and it will serve only in those cases of chronic suppuration where the attic, and particularly its outer bony wall, is unaffected.

To deal adequately with the latter type of case the simple mastoidectomy must be further extended, to expose the aditus and attic regions. This operation is called the conservative radical, antro-epitympanic, or transantral attico-tympanotomy. By it most of the contents of the middle-ear cavity can be exposed to the direct view, and lesions such as diseased ossicles, granulations, and necrosed bone removed. Proceeding further one may perform the radical mastoid operation by removing the whole of the outer attic wall, the remains of the tympanic membrane and the ossicles, and the remaining contents of the middleear cavity, respecting only the mucous lining of the inner tympanic wall, and hoping later on for epidermization of the resulting cavity. Thus we see that where the indications are not precise we can proceed conservatively, extending our operation further and further in successive steps if we consider the necessity arises as we view the extent of the lesion.

\section{Indications for Operation}

Speaking generally, however, we can often gain a fairly accurate knowledge from our clinical investigations of what type of operation will be required. These indications may now be briefly reviewed. 
1. General Indications.-Operation upon the mastoid is indicated in all cases of chronic suppuration showing evidence of threatened intracranial complication ; in those subject to acute exacerbations, complicated by facial paralysis of recent onset, or with cholesteatomatous involvement; in those which show clinically definite sagging of the post-superior meatal wall and evidence of underlying osteitis ; and in those which have resisted conservative medical treatment over a period of two to three months in adults and in children after one to two months, where any exciting cause in the nose or nasopharynx has been excluded.

Exceptions to the latter group are those in which the lesion is confined only to the atrium of the middle ear, as evidenced by the fact that the discharge is seldom more than muco-purulent, and, after the meatus is cleared, no pus can be seen on aspiration coming from the regions of the attic or antrum. These are the so-called "safe" conditions, already mentioned, with, usually, large central tympanic perforations and with hearing which is generally useful and would probably be further considerably diminished by the radical operation.

In this first group of cases it need not be necessary at all times to perferm the complete radical operation, though obviously it will be the correct procedure in cases of threatened meningeal infection originating through a diseased labyrinth, in cases of gross destruction of the middle-ear contents by cholesteatoma, or in those cases of petrositis in which the discharging peri-labyrinthine tract opens into the tympanus.

2. Early Chronic Suppuration.-The simple (or Schwartze) operation is particularly indicated in most cases of early chronic suppuration that have resisted ordinary conservative methods of treatment, especially in children, and when, as previously stated, a latent mastoiditis has been diagnosed. Also, it will probably suffice in those cases where the tympanic nembrane-especially in its superior part-does not appear unduly reddened, but where pus can be aspirated into the cleansed meatus from the antral region, the perforation being situated in the posterior portion of the tympanic membrane, even though it be central or marginal. This operation has become so classical that it would be superfluous to describe it in detail. Let me mention, however, one or two points in the technique, and also particularly as regards the aftertreatment, which in my own practice I consider of the greatest importance.

In the first place, with regard to the incision, this should be made not in the posterior auricular sulcus, as at one time advocated, but at some distance behind it, in order to allow of a curved skin-flap being raised from the underlying mastoid process, which alone it is sufficient to expose. During the healing stage this tends to avoid the possibility of extensive infection of the soft tissues or of unsightly depressions and fistulae taking place. Secondly, all diseased cells must be thoroughly opened up, especially the apical cell of the mastoid process, when the bone is of the large cellular or cancellous type. Thirdly, no plastic should be done on the meatus, and it is preferable to close the wound completely, except for the smallest of tube drainage.

A method of posterior drainage which I have adopted during the past two years in a very large number of cases, and which has given complete satisfaction as regards both the resultant healing and the minimum of discomfort to the patient (especially the infant and small child), is that of Carrel, with a solution of eusol. However, instead of cleansing the wound cavity with a continuous irrigation of eusol, it has been found more practicable and equally efficient to accomplish this by means of small injections, about $5 \mathrm{c.cm}$. at a time, made with a Record syringe through the fine tube, and repeated every one to two hours during the waking periods of the patient.
Since dressings need not be removed, except to inspect the wound occasionally, one can readily understand the ease of application of this method of after-treatment and its almost complete absence of painfulness.

3. Attic Disease.-When the disease is located in the attic region, as evidenced by the situation of a perforation in Shrapnell's membrane, or some contiguous site in the membrana, tensa, accompanied or not by a sentinel polypus, redness of the membrane in this region and the aspiration of pus from above, it is generally indicated that the attic region will have to be exposed during the mastoid operation. In such cases, especially when most of the drum membrane remains intact, the degree of useful hearing is very often found to be remarkably good. Hence we must employ an operation which, whilst dealing with the focus of suppuration, will interfere as little as possible with this hearing function.

We make use, therefore, of a type of the conservative radical operation that was first practised by Heath, and which, now modified, gives, in experienced hands, most satisfactory results. The points in the technique to which I should like to draw attention are these. A skin-flap should be turned forwards after making a curved incision extending from the upper attachment of the auricle around the hair line and thence forwards towards the tip of the mastoid process. After completing the simple mastoidectomy the next step consists in carefully separating from the bony meatus the post-superior wall of the membranous canal right down to the annulus tympanicus, where it here finds attachment to the drum membrane. The posterior bony ridge of the meatus, the outer wall of the aditus, and most of the outer attic wall are next removed. Then, by gently pushing forward the membranous meatus and the portion of drum membrane continuous and detached with it, one can now view the contents of the attic and even of the upper portion of the atrium. The body of the incus and the head of the malleus can be re-dily seen. At this stage it is essential to the success of ihe operation to make use of some form of magnifying lew or loupe, preferably of a binocular pattern, in order to essist in the location and removal of diseased membr:ane, ossicles, or bone.

If it is found necessary to remove a necrosed incus it is advisable to remove also the head of the malleus, as better drainage is thereby ensured. It must be remembered, however, that the removal of the incus unnecessarily is not to be advised if the hearing function before operation approximates to the normal, for it will certainly entail a loss of this acuity. During the first week of the aftertreatment the meatus should be firmly packed with narrow ribbon gauze, the aim being to assist the drum membrane where it has been detached from its bony support in forming a fresh attachment to the inner tympanic wall, thus creating once more a closed air space communicating only with the Eustachian tube.

4. Persistent Suppuration.-There remain after operation a fair number of cases that continue to suppurate. They may result from operation upon the acute mastoid, and may present fistulae, either posteriorly or into the meatus, stenosis of the meatus, or simply unhealed perforations with or without granulations and polypi. Others result from an incompletely epidermized radical mastoid cavity, and in the latter particularly when the tympanic orifice of the Eustachian tube remains patent. The number of such cases will, in my opinion, be considerably diminished if attention is paid to the points I have advocated-namely, a curved skin-flap incision, as little interference as possible with the membranous meatus (and this applies equally in the case of the complete radical operation), and a minimum of interference with the wound during the healing stages. If called upon to operate again 
in some of these cases only then may it become necessary for the surgeon to employ skin or muscle grafts, or some plastic form of operation on the meatus.

\section{Conclusion}

I have attempted in this paper to give a résumé of our present knowledge of the treatment of chronic middle-ear suppuration. It will be seen that we cannot claim to have made any great progress during the past decade, particularly as regards conservative measures. What, then, of the future? Upon what lines can we profitably advance, either in our medical or surgical methods? In prophylaxis there must always remain considerable scope for improvement, and much work is being done in this respect in the early observation and treatment of school children. The employment of more effective antiseptic and bactericidal solutions and powders demands further considerable research into their properties in respect to their effect upon bacteria as well as on the living tissue cell. The same applies to ionization and the various other electrical methods.

As regards surgery we, can surely devise a better technique in dealing with the problem. If, as I firmly believe, the maintenance of useful hearing depends in a lárge number of these cases upon the continued mobility of the oval and round windows, I can foresee our efforts in the future being directed towards this end, and, if fixation has occurred, even to the repair of this mechanical fault, originating surgical methods that will restore the function of the liquid within the labyrinth, thus allowing it to transmit once again the necessary correct pressure variations. Perhaps our ideal will be reached when we can graft on a new tympanic membrane, and fashion a new mobile window to replace the one that has become fixed by some adhesive or sclerotic process.

\section{THE HYPERTENSIVE CEREBRAL ATTACK} BY

DOUGLAS MCALPINE, M.D., F.R.C.P.

PHYSICIAN IN CHARGE, DEPARTMENT POR NERVOUS DISEASES, MIDDLESEX HOSPITAL; PHYSICIAN TO HOSPITAL FOR EPILEPSY AND PARALYSIS, MAIDA VALE

Until recently cerebral symptoms, such as convulsions and coma, occurring in a patient who showed raised blood pressure and albuminuria were attributed to uraemia. Volhard, ${ }^{7}$ however, pointed out that such cerebral symptoms could occur in the absence of renal insufficiency, and he used the term " pseudo-uraemia " to distinguish this condition from true uraemia. He divided pseudo-uraemia into acute and chronic forms. In the acute form there is raised intracranial pressure, and, clinically, headache, vomiting, and slowing of the pulse. These symptoms are produced by cerebral oedema, the result of an acute disturbance of the circulation in the brain. In chronic pseudo-uraemia the principal factor in the production of the cerebral symptoms is angiospasm. He believes that transient focal symptoms-such as weakness in a limb, aphasia, disturbance of vision-are caused by spasm of the corresponding cerebral vessels. In both forms a raised blood pressure is the determining factor. Oppenheimer and Fishberg ${ }^{4}$ emphasized the fact that cerebral symptoms in hypertension are generally preceded by an additional rise in blood pressure above the previous level. They give the name " hypertensive encephalopathy" to the cerebral symptoms occurring in hypertensive states. They believed that these symptoms are caused by vasoconstriction of the cerebral vessels.

It was previously held by the majority of physiologists that there was no nervous control of the cerebral blood vessels. However, the brilliant work of Forbes and Wolff $^{1}$ and their collaborators has definitely established the existence of a nervous control of the pial vessels in the cat. A summary of their experiments must suffice.

Stimulation of the cervical sympathetic in the neck by a faradic current was followed by constriction of the pial arteries. Stimulation of the vagus resulted in a dilatation of the pial arteries. They also found that the calibre of the pial vessels could be altered by changes in the chemical composition of the blood. Vaso-constriction resulted from: $(a)$ intravenous injections of hypertonic salines; $(b)$ a decrease in the carbon dioxide content, or an increase in the oxygen content of the blood; and (c) intravenous injections of pituitrin. Dilatation of these vessels followed: (1) an increase in the carbon dioxide content of the blood, or a state of anorexia ; (2) a condition of acidosis; and (3) inhalation of amyl nitrite (Wolff and Forbes, ${ }^{10}$ Wolff, ${ }^{9}$ Wolff and Lennox ${ }^{8}$ ).
Histologists such as Stöhr, ${ }^{6}$ Hassin, $^{2}$ and Penfield ${ }^{5}$ have also provided further evidence of a true nervous control of the pial vessels. They have demonstrated the existence of nerve endings in all vessels of the pia and choroid plexus. Nerve fibres have also been found in the walls of vessels entering the brain itself. These experiments and histological researches prove beyond doubt the existence of a nervous control of the superficial blood vessels of the brain, and, in all probability, of those entering the brain itself. From this it may be assumed that the blood supply of the brain is dependent on the state of tonus in the cerebral arteries and arterioles, which in its turn is under the influence of the sympathetic and autonomic nervous system. The cerebral vessels will. respond to any disturbance of the normal balance existing between these two systems, whether this be temporary or permanent, in the same manner as the blood vessels in other parts of the body, and also to changes in the chemical composition of the blood. These facts would readily explain such conditions as migraine, epilepsy, and certain of the cerebral symptoms of arterial hypertension.

\section{A Case of Pure Uraemia}

Before describing the cerebral symptoms as met with in cases of hypertension a case of pure uraemia will be described, as it affords interesting points of contrast.

A. P., a male aged 36, was admitted to the neurological ward, Middlesex Hospital, on July 26th, 1934. For a few months previously he had suffered from headaches. However, he continued at work. On July 24 th, 1934, he suddenly lost consciousness for a few minutes; the tongue was bitten, and he was incontinent. The past history was unimportant, except that in 1907 he was run over by a hansom cab and his " kidneys were damaged."

The patient had two generalized epileptiform attacks on the dày of admission, one at 3 p.m. and one at 10 p.m. Between these attacks he was conscious but drowsy. There was marked pallor of the face ; speech was dysarthric. Respirations twelve per minute; definite air-hunger. Fundi normal. No abnormal signs in the central nervous system. No muscular twitchings observed. Chvostek's sign negative. Heart: apex beat not felt, but no obvious enlargement; no added sounds. Pulse regular. Blood pressure $120 / 80$. Tongue markedly furred. Liver and spleen not enlarged. Bilateral irregular renal tumours felt. Urine: slight trace of blood; albumin, 0.07 gram per 100 c.cm.; urea, 0.57 gram per 100 c.cm. Frequent blood-pressure readings on the day of admission and the following few days showed figures ranging between $140 / 75$ and $130 / 80$. During the first three days the twenty-fourhourly output of urine varied between 49 and 57 ounces with an intake of between 62 and 87 ounces. The following blood analyses were made (figures represent milligrams per 100 c.cm.): 